\title{
Examination of the clinical usefulness of TS-1 in locally recurrent breast cancer
}

\author{
TAKEHIRO NOHARA, MITSUHIKO IWAMOTO, KAZHUHIRO SUMIYOSHI, SATORU TANAKA, \\ KOSEI KIMURA, YUKO TAKAHASHI and NOBUHIKO TANIGAWA
}

Department of General and Breast and Endocrine Surgery, Osaka Medical College, Osaka, Japan

Received March 17, 2010; Accepted May 21, 2010

DOI: 10.3892/ol_00000117

\begin{abstract}
This study examined the therapeutic efficacy of TS-1 in the treatment of locally recurrent breast cancer. Between August 2006 and May 2009, 7 patients with local breast cancer recurrences were selected included in the study. The sites of recurrence were the cervical lymph nodes in 4 patients, the axillary lymph nodes in 2 and the thoracic wall in 1 . Among the 7 patients, 6 were administered TS-1 as firstline treatment and 1 was administered TS- 1 as second-line treatment. Complete response (CR) was achieved in 2 patients, 1 achieved partial response, 2 had stable disease (SD), 1 had long SD and 1 had progressive disease. The overall response rate was $43 \%$, and the clinical benefit rate was $57 \%$. A patient with recurrence of breast cancer in the thoracic wall 28 years after surgery also achieved CR following therapy. The only adverse event observed was a case of hand-foot syndrome in 1 patient. No patients withdrew from treatment, and favorable compliance was achieved in the study. The results indicated that TS-1 has the potential to be one of the drugs for first-line treatment of locally recurrent breast cancer.
\end{abstract}

\section{Introduction}

TS-1, an oral fluoropyrimidine anti-cancer agent, contains tegafur (FT), a pro-drug of 5-fluorouracil (5-FU), as a base component, as well as two modulators of 5-FU, gimeracil and potassium oxonate (Oxo) (1). Gimeracil inhibits dihydropyrimidine dehydrogenase (DPD), an enzyme that degrades 5-FU, resulting in the maintenance of high blood levels of 5-FU. Oxo, a selective inhibitor of orotate phosphoribosyltransferase (OPRT), reduces gastrointestinal toxicity through the suppression of 5-FU activation and 5-fluoro-2'-deoxyuridine-

Correspondence to: Dr Nobuhiko Tanigawa, Department of General and Breast and Endocrine Surgery, Osaka Medical College, 2-7 Daigaku-machi, Takatuki City, Osaka 569-8686, Japan E-Mail: sur001@poh.osaka-med.ac.jp

Key words: TS-1, locally recurrent breast cancer, first-line chemotherapy 5'-monophosphate (FdUMP) synthesis in the gastrointestinal mucosa. The overall response rate of TS-1 was reported to be $21.8 \%$ in patients with recurrence of taxane-resistant breast cancer in phase II clinical studies (Hino M, et al, Proc ASCO 24: abs. 745, 2006). In late phase II clinical studies, the overall response rate of TS-1 in patients with advanced/ recurrent breast cancer was $42.0 \%$ (2). In general, primary chemotherapy recommended for recurrences of breast cancer includes administration of anthracycline and taxane (3). Oral fluoropyrimidine anti-cancer agents are less toxic (do not cause hair loss) and are easily administered to elderly patients, making the agent beneficial. Between August 2006 and May 2009, TS-1 was administered mainly as a first-line treatment to 7 patients with locally recurrent breast cancer. This study investigated TS-1 and reports the results obtained.

\section{Materials and methods}

Between August 2006 and May 2009, 7 patients with locally recurrent breast cancer were included in the study. The mean age of the patients at the time of TS-1 administration was 64 years (range 56-71). Among the patients, 1 had stage I cancer, 4 had stage II and 2 had stage III cancers. The histological type of the primary cancer was papillotubular carcinoma in 4 patients and scirrhous carcinoma in 3. Lymph node metastases were classified as $n 0$ in 3 patients, $n 1 \alpha$ in 1 and $n 2$ in 3 . A total of 4 patients with lymph node metastasis underwent postoperative chemotherapy (CAF therapy). Hormone sensitivity was found to be positive in 3 cases ( 2 cases of estrogen receptor $[\mathrm{ER}]^{+} /$progesterone receptor $[\mathrm{PgR}]^{+}$and 1 case of $\mathrm{ER}^{+} /$ $\mathrm{PgR} \mathrm{R}^{-}$) and negative in 4. Tamoxifen or aromatase inhibitors were postoperatively administered to patients with hormonesensitive tumors. Hairy-related 2 (HER2) gene expression was not observed in any patient. Although the patients showed primary recurrence, distant metastases to the lung, liver and bone were not observed at the time of TS-1 administration. TS-1 was administered as first-line treatment to 6 patients and as second-line treatment, following administration of taxane, to 1 patient. The TS-1 monotherapy was performed for all 7 patients. The sites of recurrence were the cervical lymph nodes in 4 cases, the axillary lymph nodes in 2 and the thoracic wall in 1 . The interval between tumor resection and local recurrence varied widely from 1 year and 5 months to 28 years (See Table I). 
Table I. Seven cases of locally recurrent breast cancer.

\begin{tabular}{lll}
\hline 1 & Average age & 64 years (range 56-71) \\
2 & Clinical stage & Stage I (1 case), Stage II (4 cases), Stage III (2 cases) \\
3 & Histology & Scirrhous (3 cases), Papillotubular (4 cases) \\
4 & Lymph node metastasis & n0 (3 cases), n1 $\alpha$ (1 case), n2 (3 cases) \\
5 & Pre-chemotherapy & No therapy (6 cases), docetaxel (1 case) \\
6 & Recurrent sites & Cervical lymph node (4 cases), axillary lymph node (2 cases), thoracic wall (1 case) \\
7 & Average administration of TS-1 & 9.5 cycles (range 4-18) \\
8 & Efficacy of TS-1 & CR (2 cases), PR (1 case), Long SD (1 case), SD (2 cases), PD (1 case) \\
& & Response rate 43\% (including long SD; clinical benefit, 57\%) \\
\hline
\end{tabular}

$\mathrm{CR}$, complete response; $\mathrm{PR}$, partial response; $\mathrm{SD}$, stable disease; $\mathrm{PD}$, progressive disease.

\section{3 years stage IIIA n0 (28 years after mastectomy)}

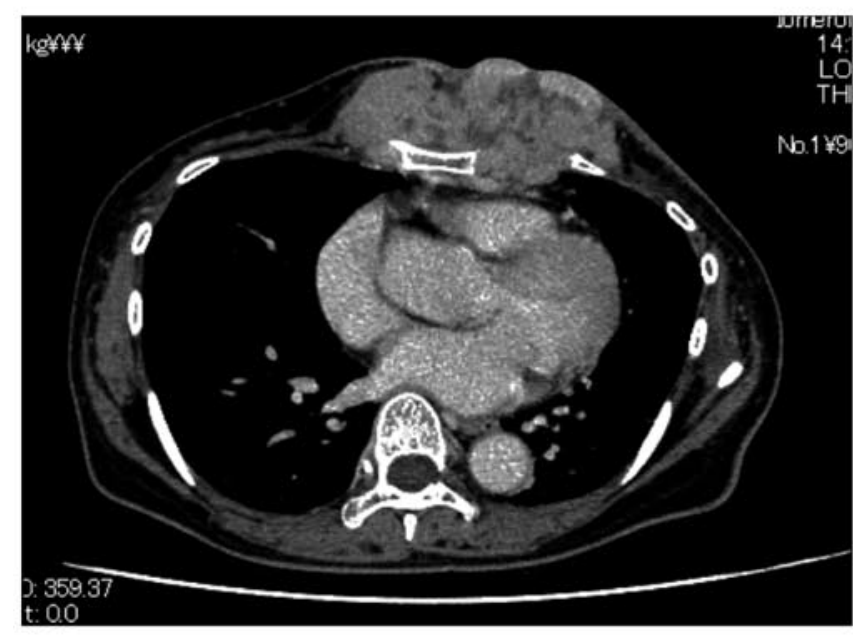

after 12 cycles of TS-1 administration (1 year and 6 months after administration )

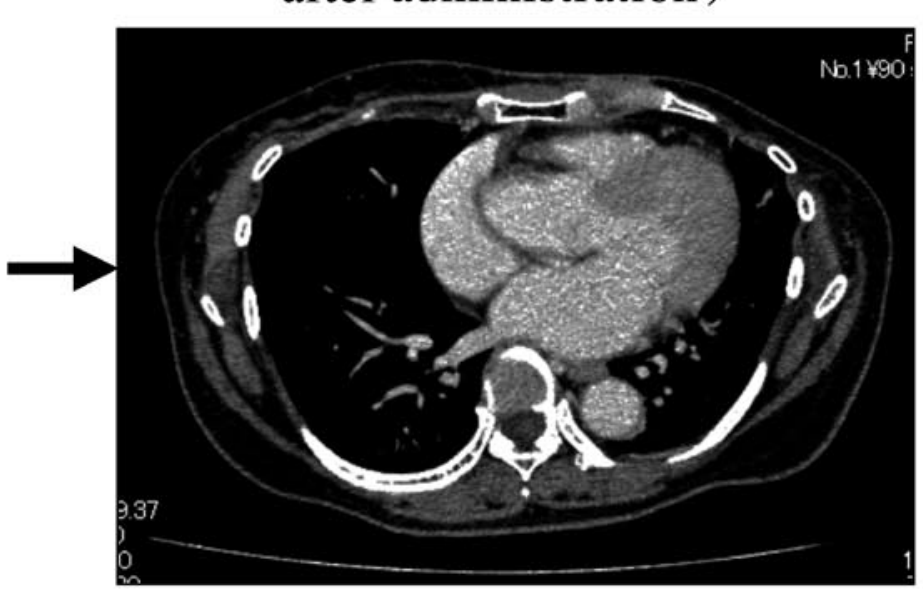

Figure 1. One case of recurrence at the thoracic wall.

Table II. A phase II study of TS-1 for advanced or recurrent breast cancer in Japan.

\begin{tabular}{|c|c|c|c|c|c|c|c|}
\hline Recurrent site & No. of patients & $\mathrm{CR}$ & PR & MR & $\mathrm{NC}$ & $\mathrm{PD}$ & Response rate $(\%)$ \\
\hline Lung & 28 & 1 & 8 & 3 & 6 & 5 & 32.1 \\
\hline Skin & 25 & 7 & 6 & 1 & 5 & 2 & 52.0 \\
\hline Liver & 6 & 0 & 1 & 0 & 2 & 1 & 16.7 \\
\hline Bone & 15 & 0 & 4 & 0 & 4 & 0 & 26.3 \\
\hline Pleura & 5 & 0 & 1 & 0 & 1 & 2 & 20.0 \\
\hline Local lymph node & 29 & 6 & 12 & 3 & 6 & 1 & 62.1 \\
\hline Visceral lymph node & 20 & 2 & 5 & 5 & 5 & 2 & 35.0 \\
\hline
\end{tabular}

$\mathrm{CR}$, complete response; PR, partial response; MR, moderate response ; NC, no change and PD, progressive disease.

The dose of TS-1 was set at $100 \mathrm{mg} /$ body/day (body surface area of $\geq 1.25 \mathrm{~m}^{2}$ and $<1.5 \mathrm{~m}^{2}$ ). Each treatment cycle lasted 6 weeks and comprised 4 weeks of consecutive administration followed by 2 weeks of off-treatment. This treatment cycle was repeated as long as the therapeutic effects were observed. However, the dose of TS-1 was reduced to $80 \mathrm{mg} /$ body/day, depending on the 
physical conditions of the patients, and continued. The average duration of TS-1 administration was 9.5 cycles (range 4-18). The efficacy of TS-1 was assessed from the 2nd or 3rd cycle after administration according to the Response Evaluation Criteria in Solid Tumors (RECIST) guidelines (4).

\section{Results}

Among the 7 patients, 2 achieved complete response (CR), 1 achieved partial response (PR), 2 had stable disease (SD), 1 had long SD and 1 had progressive disease (PD). The overall response rate was $43 \%$, and the clinical benefit rate, including long SD, was 57\% (Table I). A case in which SD was confirmed to be maintained for at least 24 weeks was judged to be long $\mathrm{SD}$ according to the RECIST guidelines. Although the time to progression (TTP) was not calculated due to the short duration of the observation periods in this study, overall local control was favorable since only 1 patient showed PD following the initial assessment of efficacy. In the CR group, 1 patient who had recurrence in the thoracic wall 28 years after surgery achieved CR after 12 cycles of TS-1 administration (Fig. 1). The mean duration of TS- 1 administration was 9.5 cycles. The status of hormone sensitivity did not affect the overall response rate. TS-1 administration resulted in hand-foot syndrome, an adverse event, in 1 case (14\%). No patients withdrew from the treatment, and the study achieved favorable compliance.

\section{Discussion}

Anthracycline and taxane are recommended for use in the first- and second-line treatment of advanced recurrent breast cancer (3). Although third-line treatment has yet to be established, oral fluoropyrimidine anti-cancer agents are often used for third-line treatment or in later treatments in patients with anthracycline- and taxane-resistant breast cancer. TS- 1 contains FT, a pro-drug of 5-FU, as a base component and combines 2 modulators, i.e., gimeracil, to inhibit DPD which is a degradation enzyme of 5-FU, and Oxo. TS-1 is different from the existing oral fluoropyrimidine anti-cancer agents as it causes less gastrointestinal toxicity and is administered in relatively higher doses (1). Capecitabine, an oral anti-cancer agent with characteristics similar to that of TS-1, was approved for use in Japan in April 2003. It has been reported that capecitabine has been considered for use up-front or as a third-line treatment (5). TS-1 was approved for use in Japan in November 2005 and was largely used as third-line treatment. The distinction between capecitabine and TS-1 for the appropriate administration in the treatment of recurrent breast cancer is a subject of further discussion. Although capecitabine was already in use up-front in other countries (6), it was not commonly administered up-front in Japan. However, a previous study suggested that up-front administration of capecitabine holds great potential for the treatment of patients with liver metastasis (5). Few studies have reported the up-front administration of TS-1 either in Japan or abroad. The results of the late phase II clinical studies conducted in Japan showed that the overall response rates of TS-1 were high in local/regional lymph nodes (62.1\%), the skin (52.0\%) and distant lymph nodes (35.0\%) (3) (Table II). This study examined the efficacy of TS-1 as a firstline treatment in locally recurrent breast cancer.
The overall response rate $(43 \%)$ and clinical benefit rate $(57 \%)$ in cases of local recurrence obtained in our hospital are consistent with those obtained in late phase II clinical studies in Japan (42 and 57\%, respectively). Although the TTP was not calculated due to the short duration of the observation periods in this study, the mean duration of administration period (9.5 cycles) was comparable to that of late phase II clinical studies in Japan (average duration of overall response was 284.5 days), as well as to a study by Yoneyama et al (7). Our study showed that the status of hormone sensitivity did not affect the overall response rate, a result that was similar to that of other late phase II clinical studies conducted in Japan (2). This study found that TS-1 is a promising drug for the effective treatment of locally recurrent breast cancer. Since oral anti-cancer agents, including capecitabine, do not induce response in cases of cancer complicated by acute and progressive diseases including lymphangitis carcinomatosa, treatment for life-threatening cases should be tailored to the needs of the patients.

TS-1, unlike other intravenously administered anti-cancer agents, causes fewer adverse reactions (hair loss and neutropenia) resulting in favorable compliance. Although 1 case of hand-foot syndrome was observed, a reduction in the dose enabled the continuation of TS-1 administration, and the patient achieved long SD. When the dose of capecitabine was reduced in order to continue administration, no differences in the TTP and overall survival were observed between the reduced and full dose (8). Therefore, TS-1, another oral anticancer drug, is anticipated to be as effective as capecitabine, even at reduced doses. In this study, favorable compliance from the patients resulted in increased therapeutic efficacy. Vitamin B6 combined with capecitabine is reported to be effective in alleviating hand-foot syndrome, an adverse reaction (9). Therefore, the concomitant administration of TS-1 with vitamin B6 is recommended. This study suggested that up-front administration of TS-1 is effective in the treatment of locally recurrent breast cancer. Taking the Hortobagyi algorism (10) into consideration, the administration of TS-1 may be one option for switching from oral anti-hormone agents with fewer adverse reactions that can be administered to elderly patients, as well as patients who have difficulties in intravenous administration and those who experience hair loss. Capecitabine has been shown to be effective in the treatment of patients with liver metastasis (5). In future, it may be possible to select either TS-1 or capecitabine for up-front administration depending on the site of tumor recurrence.

Finally, in the treatment of recurrent breast cancer, it is important not to aim to cure the cancer but to prolong the survival period while managing the symptoms and maintaining quality of life (11). The majority of cases in this study achieved either long SD or SD, resulting in a completely satisfactory outcome in terms of clinical benefit and compliance for TS-1. The combined use of TS-1 with other anti-cancer agents and antibody therapy suggests that further extension of TTP can be achieved.

\section{References}

1. Shirasaka T, Shimamoto Y, Kato T, et al: Invention of a tumorselective 5-fluorouracil derivative named S-1 by biochemical modulation of 5-fluorouracil. JPN J Cancer Chemother 25: 371-384, 1998 
2. Saeki T, Takashima S, Sano M, et al: A late phase II clinical study of S-1 in patients with progressed, refractory breast cancer. JPN J Cancer Chemother 31: 539-547, 2004.

3. The Japanese Breast Cancer Society: A Manual For EvidenceBased Clinical Practice Guideline 1 Medication. Kanehara Shuppan, Tokyo, pp91-96, 2007.

4. Therasse P, Arbuck SG, Eisenhauer EA, et al: New guidelines to evaluate the response to treatment in solid tumors. J Natl Cancer Inst 92: 205-216, 2000.

5. Nohara T, Iwamoto M, Sumiyoshi K, et al: Clinical effect and positioning of capecitabine for metastatic breast carcinoma. JPN J Cancer Chemother 35: 1315-1318, 2008.

6. William BD: Capecitabine monotherapy; safe and effective treatment for metastatic breast cancer. Oncologist 11: 325-335, 2006.

7. Yoneyama K, Yamada A, Koshida Y, et al: A patient with pulmonary metastasis from breast cancer after surgery who responded to S-1. JPN J Cancer Chemother 34: 1143-1162, 2007.
8. Shiiki S, Sonoo H, Seki M, et al: Therapeutic efficacy of capecitabine on advanced and recurrent breast cancer with special reference to time to progression. JPN J Cancer Chemother 33: 1431-1435, 2006.

9. Shigemori C, Mizutani M, Iwata $\mathrm{H}$, et al: The experience of capecitabine in advanced or reccurent breast cancer. Nyugan No Rinsyo 21: 148-152, 2006.

10. Hortobagyi GN: Treatment of breast cancer. N Engl J Med 339: 974-984, 1998.

11. Greenberg PA, Hortobagyi GN, Smith TL, et al: Long-term follow up patients with complete remission following combination chemotherapy for metastatic breast cancer. J Clin Oncol 14: 2197-2205, 1996. 